\title{
Vitiligo-Like Lesions in a Patient with Metastatic Breast Cancer Treated with Cyclin-Dependent Kinase (CDK) 4/6 Inhibitor: A Case Report and Literature Review
}

\author{
Baha' Sharaf (1D) \\ Rama AIMasri ${ }^{2}$ \\ Nayef Abdel-Razeq ${ }^{3}$ \\ Osama Salama' \\ Ibrahim Hamad (D) \\ Mahmoud Abunasser (ID) \\ Hikmat Abdel-Razeq (D) 1,4 \\ 'Department of Internal Medicine, King \\ Hussein Cancer Center, Amman, Jordan; \\ ${ }^{2}$ Department of Internal Medicine, \\ Istishari Hospital, Amman, Jordan; \\ ${ }^{3}$ Department of Internal Medicine, Henry \\ Ford Hospital, Detroit, MI, USA; ${ }^{4}$ School \\ of Medicine, University of Jordan, \\ Amman, Jordan
}

Background: Cyclin-dependent kinase (CDK) 4/6 inhibitors have revolutionized the treatment landscape of hormone receptor-positive $\left(\mathrm{HR}^{+}\right)$, human epidermal growth factor receptor 2-negative $\left(\mathrm{HER}^{-}\right)$metastatic breast cancer, with an impressive efficacy and safety profile. Cytopenia is the main adverse event, which is both predictable and manageable. Here, we report a case of CDK4/6 inhibitor-induced vitiligo-like lesions. Vitiligo or vitiligolike lesions are a rare adverse event; only a few cases are reported in the literature.

Case Presentation: A 71-year-old female patient was diagnosed initially with early-stage right breast cancer $\left(\mathrm{HR}^{+} / \mathrm{HER} 2^{-}\right)$and was treated with breast-conserving surgery followed by chemotherapy, radiotherapy, and hormonal therapy. A few years later, she developed metastatic disease to the hilar lymph nodes, and to multiple skeletal sites, including the left scapula, left shoulder, left iliac bone, and dorsal vertebrae, for which she was treated with ribociclib and letrozole. While on treatment, she developed hypopigmented lesions involving both hands, feet, and face, which were described as vitiligo-like lesions.

Conclusion: CDK4/6 inhibitor-induced vitiligo is a rare and unpredictable adverse event. This case report highlights the rarity of this adverse event, the dilemma related to the optimal treatment, and decisions related to continuation, holding, or switching CDK4/6 inhibitors.

Keywords: CDK4/6 inhibitors, ribociclib, vitiligo, vitiligo-like, breast cancer, skin adverse events

\section{Introduction}

The cyclin-dependent kinase (CDK) 4/6 inhibitors are novel targeted therapeutic agents that interrupt the proliferation of malignant cells through the inhibition of cyclin-D/ CDK4/6 complex activity, so that retinoblastoma protein remains hypophosphorylated and bound to E2F transcription factor and, as a result, blocks cell cycle progression from G1 to S phase. ${ }^{1,2}$ These drugs, combined with aromatase inhibitors or fulvestrant, have revolutionized the treatment landscape of hormone receptor-positive $\left(\mathrm{HR}^{+}\right)$, human epidermal growth factor receptor 2-negative (HER2 ${ }^{-}$) metastatic breast cancer $(\mathrm{mBC})$. They have provided consistent and impressive efficacy outcomes in various settings, and combination regimens in first line treatment and beyond, for both premenopausal and postmenopausal women. ${ }^{3-7}$

All three available CDK 4/6 inhibitors - palbociclib, ribociclib, and abemaciclib - have shown comparable efficacy outcomes, with variable, but predictable and
Correspondence: Hikmat Abdel-Razeq Department of Internal Medicine, King Hussein Cancer Center, School of Medicine, University of Jordan, Queen Rania Al Abdullah Street, PO Box 1269, Amman II 94I, Jordan Tel + 962-6 5300460, Ext: 1000 Email habdelrazeq@khcc.jo 
manageable adverse events. ${ }^{8}$ In addition to improving the time to disease progression, and possibly improving overall survival, ${ }^{9}$ quality of life (QoL) is an important outcome indicator, which is maintained or improved across all CDK4/6 inhibitor trials. ${ }^{10-13}$

Bone marrow myelosuppression, mainly neutropenia, is the most commonly encountered adverse event, and is probably a class effect, reported in almost $80 \%$ of the studied patients in all clinical trials involving all three CDK4/6 inhibitors. ${ }^{14-16}$

A number of mild cutaneous adverse events, such as low-grade alopecia and skin rash, have been attributed to CDK $4 / 6$ inhibitors. ${ }^{17}$ More severe skin toxicities are rare and constitute less than $1 \%$ of reported adverse events. ${ }^{18,19}$ Vitiligo is a chronic acquired pigmentary skin disorder, characterized by the absence of pigmentary cells from the epidermis that leads to white patches on the body, usually symmetrically distributed, and is more obvious in people with dark skin. The lesions are mostly well-demarcated white patches in different shapes, and they vary in size from a few millimeters to centimeters. The initial lesions are most frequently encountered on the hands, feet, forearms, and face, favoring a periocular or perioral distribution. The etiology of vitiligo is unknown, but it is usually associated with autoimmune disorders, mostly thyroid diseases. Because of the uncertainty of its etiology, its natural course and the absence of effective treatment, there is a sense of social stigmatization, psychological distress, and low self-esteem among affected patients.

Vitiligo or vitiligo-like lesions are rarely reported with CDK4/6 inhibitors. $^{20}$ It is proposed that this adverse event may be due to the apoptotic effect of CDK4/6 inhibitors on melanocytes, which may result in reduced numbers and survival of melanocytes, and, consequently, the appearance of hypopigmented lesions. ${ }^{21}$

\section{Methods}

Following the identification of our case, all published clinical trials, case reports, reviews, and meta-analyses on the use of CDK4/6 inhibitors, plus aromatase inhibitors or fulvestrant, were reviewed for reported adverse events. The literature review was conducted utilizing PubMed, with the following keywords: CDK4/6 inhibitors, ribociclib, palbociclib, abemaciclib, vitiligo, vitiligo-like lesions, breast cancer, and skin adverse events.

\section{Case Report}

A 71-year-old female patient with thalassemia minor and hypertension was diagnosed in 1999 with early-stage right breast cancer. She underwent breast-conserving surgery and axillary dissection; pathology showed a small tumor, less than $2 \mathrm{~cm}$, node-negative invasive ductal carcinoma. Hormone receptors (HRs) were positive but HER2 was negative. After the surgery, she received chemotherapy [cyclophosphamide, methotrexate, and 5-fluorouracil (CMF)] followed by radiotherapy and adjuvant tamoxifen for 5 years. She did well and was very adherent to clinical and mammographic follow-up until 2015, when she had an intractable cough. Imaging studies showed a left hilar lesion and multiple osteolytic bone metastases, including the left scapula, left shoulder, left iliac bone, and the fourth and seventh dorsal vertebrae. Fine needle aspiration from the left hilum, through endobronchial ultrasound, confirmed breast cancer metastasis. Similarly to the original tumor, hormone receptors were positive (ER 90\%, PR 30\%) and HER2 was negative. Therefore, she was started on endocrine therapy with fulvestrant, which kept her progression free for 2 years. She was then switched to exemestane and everolimus; however, this regimen was discontinued owing to grade II pneumonitis and prenephrotic range proteinuria. At that time, the patient was enrolled in a double-blind randomized clinical trial testing CDK4/6 inhibitors along with aromatase inhibitors. A few months later, the patient was taken off the study owing to disease progression, and was found to be on the placebo arm. Following the US Food and Drug Administration (FDA) approval of CDK4/6 inhibitors, she was started on ribociclib and letrozole, which she tolerated relatively well. Nonetheless, because of persistent neutropenia, the dose of ribociclib was reduced to $400 \mathrm{mg}$ daily; on days 1-21 of a 28-day cycle.

Almost 20 weeks after starting ribociclib and letrozole, she noticed small, rounded, well-demarcated hypopigmented lesions over both hands with expanding patches reaching up to the mid forearms (Figure 1). Three weeks later, new similar lesions started to appear over her face (Figure 2) and feet. The patient had no personal or family history of autoimmune diseases. The patient was seen at the dermatology clinic and was offered treatment with topical immunomodulators (calcineurin inhibitors), which she refused, and was kept on topical steroids with little improvement. 


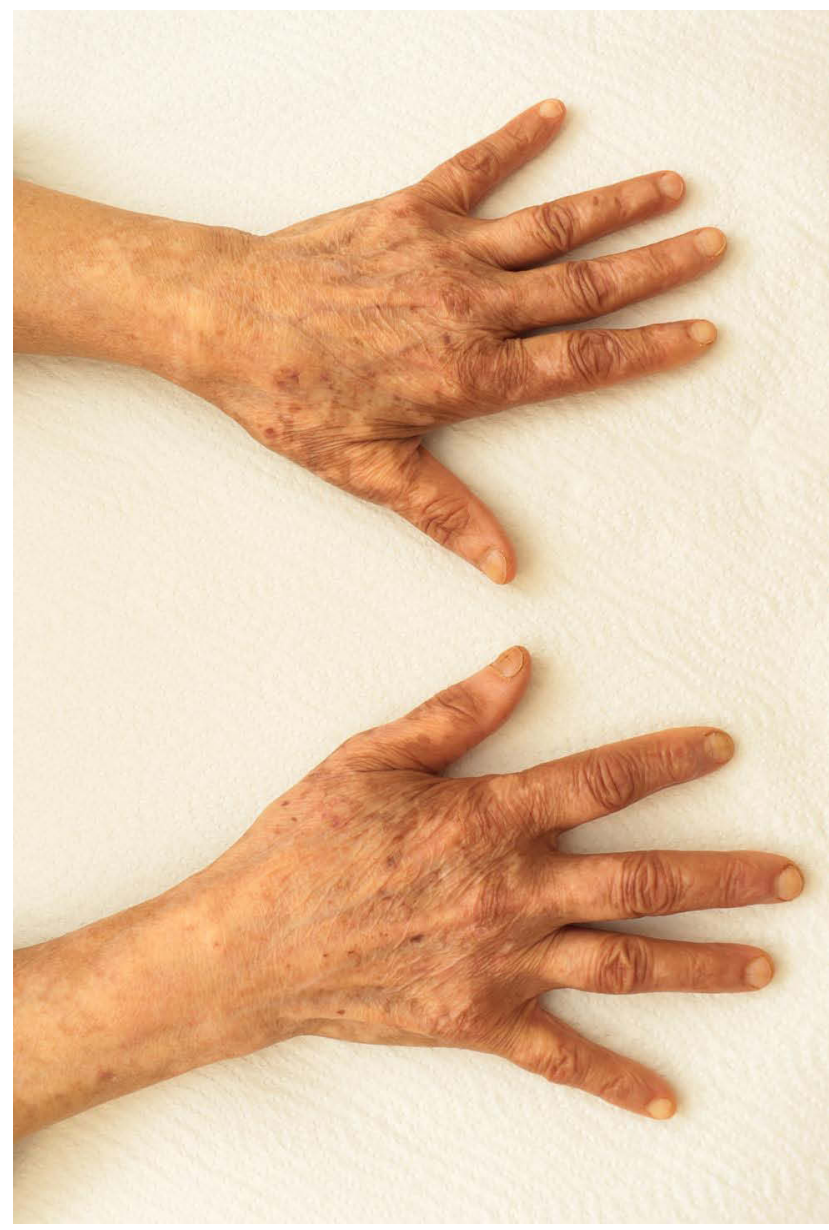

Figure I Well-demarcated, hypopigmented lesions over the hands with expanding patches reaching up to the forearms.

\section{Discussion}

Cutaneous adverse events can be a major problem, and may directly impact the QoL of patients receiving anticancer therapy. ${ }^{22,23} \mathrm{~A}$ systematic review and meta-analysis reviewed the risk and incidence of pigmentary lesions, as adverse events, among 8052 patients treated with targeted therapy and enrolled in 36 clinical trials. In total, $17.7 \%$ of patients had depigmented skin lesions. ${ }^{24}$ Imatinib, cabozantinib, nivolumab, pazopanib, pembrolizumab, sorafenib, and sunitinib appeared to be the most common culprits.

A wide range of dermatological manifestations, of varying degree, were reported with CDK4/6 inhibitors (Table 1). Alopecia, for example, was reported in $33.2 \%$ of patients treated with the combination of ribociclib and letrozole, compared to $15.5 \%$ treated with letrozole alone, in the MONALEESA-2 trial; and $14.8 \%$ with palbociclib and fulvestrant, compared to $5.8 \%$ with fulvestrant alone, in the PALOMA-3 trial. ${ }^{25}$ More severe and devastating

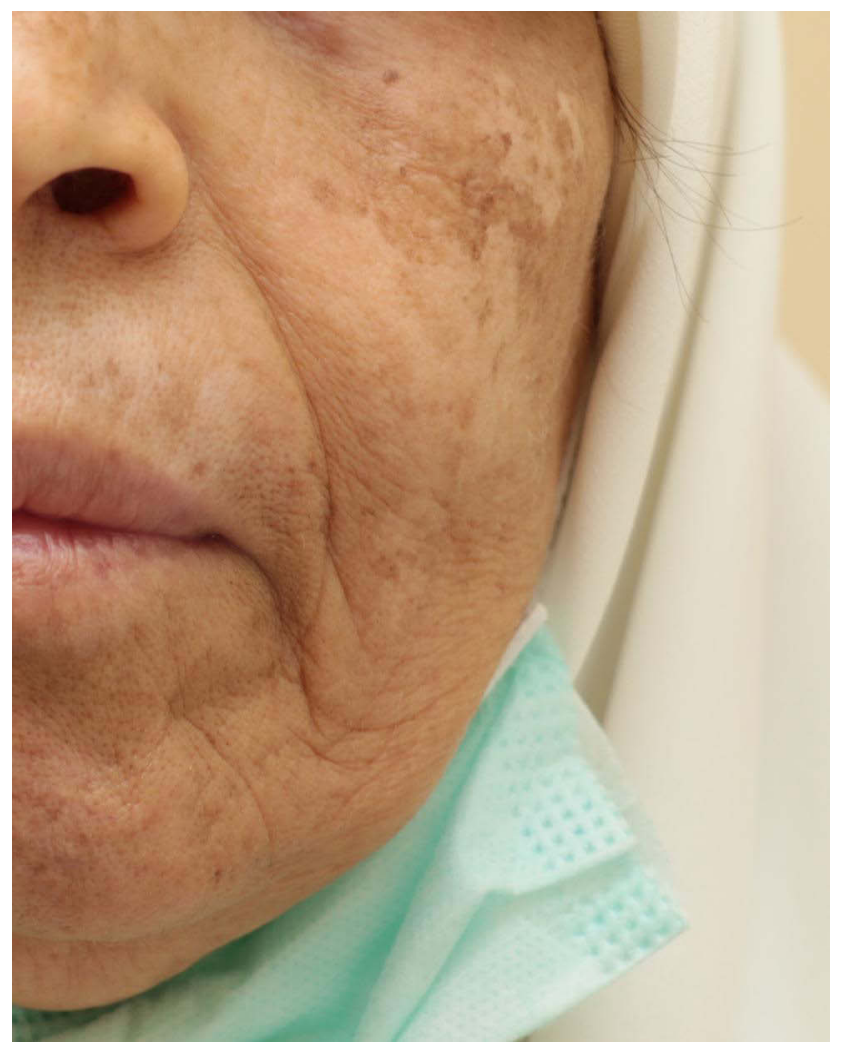

Figure 2 Vitiligo-like lesions over the face. Depigmented patches surrounded by normal skin. The patches are whitish in color and are well demarcated.

events have been reported; one case of acute bullous skin rash, $^{26}$ three cases of Stevens-Johnson syndrome (SJS), ${ }^{18,27,28}$ one case of toxic epidermal necrolysis (TEN), ${ }^{29}$ one case of histiocytoid Sweet syndrome, ${ }^{30}$ and one case of erythema dyschromicum perstans (ashy dermatosis)-like pigmentation. ${ }^{31}$

The association between cancer and vitiligo has been previously studied. The risk of melanoma and nonmelanoma skin cancers was significantly lower in a group of 10,040 Italian patients with vitiligo. ${ }^{32}$ Several other studies reached similar conclusions, and similar trends were also seen with internal organ carcinomas. $^{33,34}$ In a retrospective Korean study from

Table I Dermatological Manifestations Reported with CDK4/6 Inhibitors

- Alopecia

- Vitiligo

- Acute bullous skin rash

- Stevens-Johnson syndrome (SJS)

- Toxic epidermal necrolysis (TEN)

- Histiocytoid Sweet syndrome 
2019, which included 101,078 patients with vitiligo and 202,156 controls, the incidence of "internal" cancers was 612.9 per 100,000 person-years in patients with vitiligo, compared to 708.9 per 100,000 person-years (HR, 0.86; $P<0.001)$ among the controls. ${ }^{35}$ It is hypothesized that patients with autoimmune diseases, such as vitiligo, have higher immune surveillance, which, in turn, targets cancer development. In contrast, many previous studies had shown an increased risk of internal malignancy with other autoimmune diseases, such as systemic lupus and rheumatoid arthritis. ${ }^{36-39}$ These conflicting results may be explained by the different treatment modalities of vitiligo and other autoimmune diseases.

The increase in the use of immunotherapy to treat various cancers has led to more attention being paid to vitiligo. The appearance of vitiligo among cancer patients on immunotherapy has been associated with favorable outcomes in melanoma ${ }^{40,41}$ and renal cell carcinoma. ${ }^{42}$ However, with the limited number of vitiligo or vitiligolike cases reported after exposure to CDK4/6 inhibitors, similar conclusions cannot be reached.

To our knowledge, only 18 previous cases of CDK4/6 inhibitor-induced vitiligo-like lesions have been reported in the literature. Sollena et al, on behalf of the European Network for Cutaneous ADverse event to Oncologic drugs (ENCADO) group, reported the largest series of vitiligo-like lesions associated with $\mathrm{CDK} 4 / 6$ inhibitors used in the treatment of 16 patients with $\mathrm{mBC}$ at six European university-based centers. ${ }^{20}$ The majority of the patients were treated with topical steroids and/or topical calcineurin inhibitors, with variable response. The two other cases were reported by Chan et al, in 71- and 54-year-old women; both of them were treated with ribociclib and letrozole and neither had a personal or familial history of autoimmune diseases. ${ }^{43}$ Our case should add to the knowledge gained from existing case reports in the literature. First, vitiligo-like skin lesions may be encountered somewhat late during the course of ribociclib therapy. Second, a significant response to the usual vitiligo therapy may not occur, yet continuation of ribociclib is possible as long as patients are willing to carry on taking it.

We believe that the incidence of ribociclib, and probably the other CDK4/6 inhibitor-induced cutaneous adverse events, especially vitiligo or vitiligo-like lesions, is underreported. Physicians tend to pay more attention to the more serious and dose-limiting toxicities, such as cytopenia, cardiac dysfunction, and hepatotoxicity.

With the increasing utilization of CDK4/6 inhibitors in the most common subset of $\mathrm{mBC}\left(\mathrm{HR}^{+} / \mathrm{HER} 2^{-}\right)$, and their recent approval in HER2-positive disease, ${ }^{44}$ in addition to the recent efforts to move their use to earlier stages of breast cancer, ${ }^{45} \mathrm{CDK} 4 / 6$ inhibitor-induced vitiligo-like lesions will probably be more commonly encountered.

\section{Conclusions}

Ribociclib-induced vitiligo-like lesions are rare, but probably underreported. This case report highlights the rarity of this adverse event, the dilemma related to the optimal treatment, and the difficult decisions related to continuation, holding, or switching to other CDK4/6 inhibitors.

\section{Ethical Statement}

Written informed consent was provided by the patient to have the case details and accompanying images published. Permission to publish this case report was also obtained from the Institutional Review Board (IRB) at King Hussein Cancer Center.

\section{Acknowledgment}

The authors would like to acknowledge the efforts of our medical photographers, Mr. Ameen Harb and Mr. Ramzi Abu-Khader.

\section{Disclosure}

The authors declare no conflicts of interest in this work.

\section{References}

1. Sammons S, Topping D, Blackwell K. HR+, HER2- advanced breast cancer and CDK4/6 inhibitors: mode of action, clinical activity, and safety profiles. Curr Cancer Drug Targets. 2017;17(7). doi:10.2174/ 1568009617666170330120452

2. O'Leary B, Finn RS, Turner NC. Treating cancer with selective CDK4/6 inhibitors. Nat Rev Clin Oncol. 2016;13(7):417-430. doi:10.1038/nrclinonc.2016.26

3. Finn RS, Martin M, Rugo HS, et al. Palbociclib and letrozole in advanced breast cancer. $N$ Engl J Med. 2016;375(20):1925-1936. doi:10.1056/NEJMoa1607303

4. Cristofanilli M, Turner NC, Bondarenko I, et al. Fulvestrant plus palbociclib versus fulvestrant plus placebo for treatment of hormone-receptor-positive, HER2-negative metastatic breast cancer that progressed on previous endocrine therapy (PALOMA-3): final analysis of the multicentre, double-blind, Phase 3 randomised controlled trial. Lancet Oncol. 2016;17:425-439. doi:10.1016/S14702045(15)00613-0.

5. Tripathy D, Im S-A, Colleoni M, et al. Ribociclib plus endocrine therapy for premenopausal women with hormone-receptor-positive, Advanced Breast Cancer (MONALEESA-7): a randomised phase 3 trial. Lancet Oncol. 2018;19(7):904-915. doi:10.1016/s1470-2045(18) 30292-4

6. Sledge GW, Toi M, Neven P, et al. Monarch 2: abemaciclib in combination with fulvestrant in women with HR+/HER2- advanced breast cancer who had progressed while receiving endocrine therapy. J Clin Oncol. 2017;35(25):2875-2884. doi:10.1200/jco.2017.73.7585 
7. Hortobagyi GN. Ribociclib for the first-line treatment of advanced hormone receptor-positive breast cancer: a review of subgroup analyses from the MONALEESA-2 trial. Breast Cancer Res. 2018;20:1. doi:10.1186/s13058-018-1050-7

8. Desnoyers A, Nadler MB, Kumar V, Saleh R, Amir E. Comparison of treatment-related adverse events of different cyclin-dependent kinase 4/6 inhibitors in metastatic breast cancer: a network meta-analysis. Cancer Treat Rev. 2020;90:102086. doi:10.1016/j.ctrv.2020.102086

9. Spring LM, Wander SA, Andre F, Moy B, Turner NC, Bardia A. Cyclin-dependent kinase 4 and 6 inhibitors for hormone receptor-positive breast cancer: past, present, and future. Lancet. 2020;395(10226):817-827. doi:10.1016/s0140-6736(20)30165-3

10. Harbeck N, Iyer S, Turner N, et al. Quality of life with palbociclib plus fulvestrant in previously treated hormone receptor-positive, HER2-negative metastatic breast cancer: patient-reported outcomes from the paloma-3 trial. Ann Oncol. 2016;27(6):1047-1054. doi:10.1093/annonc/mdw139

11. Verma S, O'Shaughnessy J, Burris HA, et al. Health-related quality of life of postmenopausal women with hormone receptor-positive, human epidermal growth factor receptor 2-negative advanced breast cancer treated with ribociclib + letrozole: results from monaleesa-2. Breast Cancer Res Treat. 2018;170(3):535-545. doi:10.1007/s10549018-4769-z

12. Kaufman PA, Toi M, Neven P, et al. Health-related quality of life in MONARCH 2: abemaciclib plus fulvestrant in hormone receptor-positive, her2-negative advanced breast cancer after endocrine therapy. Oncologist. 2020;25(2):e243-e251. doi:10.1634/theoncologist.2019-0551

13. Fasching PA, Esteva FJ, Pivot X, et al. Patient-reported outcomes (pros) in advanced breast cancer $(\mathrm{ABC})$ treated with ribociclib + fulvestrant: results from monaleesa-3. Ann Oncol. 2018;29:viii90. doi:10.1093/annonc/mdy272.282

14. Yang L, Xue J, Yang Z, et al. Side effects of CDK4/6 inhibitors in the treatment of HR+/HER2- advanced breast cancer: a systematic review and meta-analysis of randomized controlled trials. Ann Palliat Med. 2021;10(5):5590-5599. doi:10.21037/apm-21-1096

15. Messina C, Cattrini C, Buzzatti G, et al. CDK4/6 inhibitors in advanced hormone receptor-positive/HER2-negative breast cancer: a systematic review and meta-analysis of randomized trials. Breast Cancer Res Treat. 2018;172(1):9-21. doi:10.1007/s10549-018-4901-0

16. Messina C, Messina M, Zanardi E. Risks and benefits from CDK inhibitors for advanced HR+, HER2- breast cancer. Ann Oncol. 2017;28(12):3099-3100. doi:10.1093/annonc/mdx530 PMID: 28950333.

17. Eiger D, Wagner M, Pondé NF, Nogueira MS, Buisseret L, de Azambuja E. The impact of cyclin-dependent kinase 4 and 6 inhibitors $(\mathrm{cdk} 4 / 6 \mathrm{i})$ on the incidence of alopecia in patients with Metastatic Breast Cancer (BC). Acta Oncol. 2020;59(6):723-725. doi:10.1080/ 0284186x.2020.1738547

18. Karagounis T, Vallurupalli M, Nathan N, et al. Stevens-Johnson syndrome-like eruption from palbociclib in a patient with metastatic breast cancer. JAAD Case Rep. 2018;4(5):452-454. doi:10.1016/j. jdcr.2017.12.015

19. Silvestri M, Cristaudo A, Morrone A, et al. Emerging skin toxicities in patients with breast cancer treated with new cyclin-dependent kinase 4/6 inhibitors: a systematic review. Drug Saf. 2021;44 (7):725-732. doi:10.1007/s40264-021-01071-1

20. Sollena P, Nikolaou V, Soupos N, et al. European Network for Cutaneous ADverse event of Oncologic drugs (ENCADO) group. Vitiligo-like lesions in patients with advanced breast cancer treated with cycline-dependent kinases 4 and 6 inhibitors. Breast Cancer Res Treat. 2021;185(1):247-253. doi:10.1007/s10549020-05914-w

21. Zhou M, Lin F, Xu W, Jin R, Xu A. Decreased sumoylation of the retinoblastoma protein in keratinocytes during the pathogenesis of Vitiligo. Mol Med Rep. 2018. doi:10.3892/mmr.2018.9299
22. Hoffmann T, Corrêa-Fissmer M, Duarte CS, Nazário RF, Barranco AB, Oliveira KW. Prevalence of dermatological complaints in patients undergoing treatment for breast cancer. An Bras Dermatol. 2018;93(3):362-367. doi:10.1590/abd1806-4841.20186541

23. Balagula Y, Rosen ST, Lacouture ME. The emergence of supportive oncodermatology: the study of Dermatologic Adverse Events To Cancer Therapies. J Am Acad Dermatol. 2011;65(3):624-635. doi:10.1016/j.jaad.2010.06.051

24. Dai J, Belum VR, Wu S, Sibaud V, Lacouture ME. Pigmentary changes in patients treated with targeted anticancer agents: a systematic review and meta-analysis. $J$ Am Acad Dermatol. 2017;77:5. doi:10.1016/j.jaad.2017.06.044

25. Saggar V, Wu S, Dickler MN, Lacouture ME. Alopecia with endocrine therapies in patients with cancer. Oncologist. 2013;18 (10):1126-1134. doi:10.1634/theoncologist.2013-0193

26. Khan NA, Alsharedi M. Bullous skin rash: a rare case of palbociclib-induced dermatological toxicity. Cureus. 2020. doi:10.7759/cureus. 10229

27. Widmer S, Grossman M. Chemotherapy patient with Stevens-Johnson Syndrome presents to the Emergency Department: a case report. Am J Emerg Med. 2018;36:7. doi:10.1016/j.ajem.20 18.04 .019

28. López-Gómez V, Yarza R, Muñoz-González H, et al. Ribociclibrelated Stevens-Johnson syndrome: oncologic awareness, case report, and literature review. J Breast Cancer. 2019;22(4):661. doi:10.4048/ jbc.2019.22.e44

29. Bozkaya Y, Ay B. Toxic epidermal necrolysis associated with ribociclib in a patient with metastatic breast cancer. Breast J. 2019;26 (4):773-774. doi:10.1111/tbj.13570

30. Fustà-Novell X, Morgado-Carrasco D, García-Herrera A, BoschAmate X, Martí-Martí I, Carrera C. Palbociclib-induced histiocytoid sweet syndrome. Clin Exp Dermatol. 2020;46(2):348-350. doi:10.1111/ced.14361

31. Mariano M, Donati P, Cameli N, Pigliacelli F, Morrone A, Cristaudo A. Ribociclib-induced erythema dyschromicum perstans (Ashy Dermatosis)-like pigmentation in a metastatic breast cancer patient. J Breast Cancer. 2021;24(1):117-121. doi:10.4048/jbc.20 21.24.e1

32. Paradisi A, Tabolli S, Didona B, Sobrino L, Russo N, Abeni D. Markedly reduced incidence of melanoma and nonmelanoma skin cancer in a nonconcurrent cohort of 10,040 patients with Vitiligo. J Am Acad Dermatol. 2014;71(6):1110-1116. doi:10.1016/j.jaad. 2014.07.050

33. Teulings HE, Overkamp M, Ceylan E, et al. Decreased risk of melanoma and nonmelanoma skin cancer in patients with Vitiligo: a survey among 1307 patients and their partners. Br J Dermatol. 2012;168(1):162-171. doi:10.1111/bjd.12111

34. Schallreuter KU, Tobin DJ, Panske A. Decreased photodamage and low incidence of non-melanoma skin cancer in 136 sun-exposed Caucasian patients with Vitiligo. Dermatology. 2002;204(3):19 4-201. doi:10.1159/000057881

35. Bae JM, Chung KY, Yun SJ, et al. Markedly reduced risk of internal malignancies in patients with vitiligo: a nationwide population-based Cohort Study. J Clin Oncol. 2019;37(11):903-911. doi:10.1200/jco. 18.01223

36. Hemminki K, Li X, Sundquist K, Sundquist J. Cancer risk in hospitalized rheumatoid arthritis patients. Rheumatology. 2008;47 (5):698-701. doi:10.1093/rheumatology/ken130

37. Ni J, Qiu L-J, Hu L-F, et al. Lung, liver, prostate, bladder malignancies risk in systemic lupus erythematosus: evidence from a meta-analysis. Lupus. 2014;23(3):284-292. doi:10.1177/09612033 13520060

38. Simon TA, Thompson A, Gandhi KK, Hochberg MC, Suissa S. Incidence of malignancy in adult patients with rheumatoid arthritis: a meta-analysis. Arthritis Res Ther. 2015;17:1. doi:10.1186/s13075015-0728-9 
39. Wang H-L, Zhou Y-M, Zhu G-Z, Yang Z, Hua B-J. Malignancy as a comorbidity in rheumatic diseases: a retrospective hospital-based study. Clin Rheumatol. 2017;37(1):81-85. doi:10.1007/s10067-0173676-8

40. Teulings H-E, Limpens J, Jansen SN, et al. Vitiligo-like depigmentation in patients with stage III-IV melanoma receiving immunotherapy and its association with survival: a systematic review and meta-analysis. J Clin Oncol. 2015;33(7):773-781. doi:10.1200/ jco.2014.57.4756

41. Hua C, Boussemart L, Mateus C, et al. Association of vitiligo with tumor response in patients with metastatic melanoma treated with Pembrolizumab. JAMA Dermatol. 2016;152(1):45. doi:10.1001/ jamadermatol.2015.2707
42. Billon E, Walz J, Brunelle S, et al. Vitiligo adverse event observed in a patient with durable complete response after nivolumab for metastatic renal cell carcinoma. Front Oncol. 2019;9. doi:10.3389/ fonc. 2019.01033

43. Chan OB, Su JC, Yazdabadi A, Chan A. Drug induced vitiligo-like depigmentation from a CDK 4/6 inhibitor. Asia Pac J Clin Oncol. 2021. doi:10.1111/ajco. 13585

44. O'Sullivan CC, Suman VJ, Goetz MP. The emerging role of cdk4/6i in HER2-positive breast cancer. Ther Adv Med Oncol. 2019;11:175883591988766. doi:10.1177/1758835919887665

45. Gil-Gil M, Alba E, Gavilá J, et al. The role of CDK4/6 inhibitors in early breast cancer. Breast. 2021;58:160-169. doi:10.1016/j. breast.2021.05.008

\section{Publish your work in this journal}

Clinical, Cosmetic and Investigational Dermatology is an international, peer-reviewed, open access, online journal that focuses on the latest clinical and experimental research in all aspects of skin disease and cosmetic interventions. This journal is indexed on CAS.
The manuscript management system is completely online and includes a very quick and fair peer-review system, which is all easy to use. Visit http://www.dovepress.com/testimonials.php to read real quotes from published authors. 